\section{Long-term Oncologic Outcomes of I 88 Tis-T2 Glottic Cancers Treated by Transoral Laser Microsurgery}

\author{
Cesare Piazza, MD', Alberto Paderno, MD', \\ Francesca Del Bon, MD', Davide Lancini, MD', Milena Fior, MD', \\ Giulia Berretti, MD', Paolo Bosio, MD', Alberto Deganello, PhD', \\ and Giorgio Peretti, MD $^{2}$
}

Otolaryngology-

Head and Neck Surgery

$1-8$

(C) American Academy of

Otolaryngology-Head and Neck

Surgery Foundation 2021

Reprints and permission:

sagepub.com/journalsPermissions.nav DOI: $10.1177 / 0194599820983727$ http://otojournal.org

(S)AGE

\begin{abstract}
Objective. To evaluate long-term disease-specific survival (DSS) and organ preservation (OP) rates in patients affected by Tis-T2 glottic squamous cell carcinoma (SCC) treated by carbon dioxide transoral laser microsurgery $\left(\mathrm{CO}_{2} \mathrm{TOLMS}\right)$.
\end{abstract}

Study Design. Single-center retrospective cohort study.

Setting. Tertiary academic hospital.

Methods. The study included patients treated by $\mathrm{CO}_{2}$ TOLMS for Tis-T2 glottic SCC at the Department of Otorhinolaryngology-Head and Neck Surgery of the University of Brescia, Italy, from 1988 to 2018. The male:female ratio was II.2:I, and the mean age was 64 years (range, 31-95). T categories were distributed as follows: 124 (I0\%) Tis, 646 (54\%) TIa, I72 (15\%) TIb, and 246 (2I\%) T2.

Results. Ten- and 20-year DSS rates were $97.6 \%$ and $96.3 \%$, respectively, and 10- and 20-year OP rates were $94.7 \%$ and $93 \%$. During the follow-up, $91 \%$ of patients were treated by $\mathrm{CO}_{2}$ TOLMS alone, while the remaining needed adjunctive treatments. Assessing the impact of multiple sessions of $\mathrm{CO}_{2}$ TOLMS, DSS showed no significant difference in terms of patients treated by I, 2 , or $>2$ procedures. Conversely, patients treated by $>2$ sessions of $\mathrm{CO}_{2}$ TOLMS showed a significantly worse OP rate.

Conclusions. Our series validates $\mathrm{CO}_{2}$ TOLMS as a long-term treatment strategy for early glottic SCC. Salvage $\mathrm{CO}_{2}$ TOLMS provided optimal results in terms of DSS and OP in patients with recurrence after previous transoral surgery.

\section{Keywords}

transoral laser microsurgery, laryngeal cancer, glottic, early, long-term, oncologic outcomes

Received September 29, 2020; accepted December 5, 2020.

arbon dioxide transoral laser microsurgery $\left(\mathrm{CO}_{2}\right.$ TOLMS), as recently classified by Remacle and coworkers, ${ }^{1}$ is an established treatment option for early-intermediate glottic squamous cell carcinoma (EIGSCC). However, its indications are highly variable and influenced mainly by institution- and country-based policies. Furthermore, decades after its introduction and implementation, high-quality data are still lacking confirming the value of $\mathrm{CO}_{2}$ TOLMS as a therapeutic tool and its results in comparison with alternative options, such as radiotherapy (RT) and open partial horizontal laryngectomies (OPHLs), ${ }^{2}$ especially in terms of long-term follow-up. The Cochrane Collaboration regularly updates a systematic review and meta-analysis aimed at assessing $\mathrm{CO}_{2}$ TOLMS, RT, and partial laryngectomies for EIGSCC, including all prospective randomized trials on this topic. ${ }^{3}$ However, the only study selected by the reviewers to date did not include patients treated by $\mathrm{CO}_{2}$ TOLMS, and it was classified as being at high risk of bias. ${ }^{4}$ For this reason, the comparison was limited to RT and partial laryngectomies.

However, high-quality data are essential to better assess surgical innovations and direct their development. The rationale is that if the opportunity for robust evaluation is not seized, widespread adoption of a given therapeutic tool may take place without adequate evidence. This is particularly true in settings where randomized clinical trials are not easily feasible due to technical constraints, ethical concerns, and patient preference. In this context, evidence should arise from high-quality nonrandomized prospective or retrospective studies specifically assessing each aspect of the technique. In particular, long-term outcomes represent one of the main measures to be considered.

The aim of our study was therefore to retrospectively evaluate long-term disease-specific survival (DSS) and organ

\footnotetext{
'Department of Otorhinolaryngology-Head and Neck Surgery, University of Brescia, ASST-Spedali Civili of Brescia, Brescia, Italy

${ }^{2}$ Department of Otorhinolaryngology-Head and Neck Surgery, University of Genoa, IRCCS Ospedale Policlinico San Martino, Genoa, Italy

This article was accepted as "Best of Orals" at the AAO-HNSF 2020 Virtual Annual Meeting \& OTO Experience; September I3-October 25, 2020.

Corresponding Author:

Alberto Paderno, MD, Department of Otorhinolaryngology-Head and Neck Surgery, University of Brescia, ASST-Spedali Civili of Brescia, 25I23 Brescia, Italy.

Email: albpaderno@gmail.com
} 
preservation (OP) rates in a large homogeneous cohort of patients affected by Tis-T2 glottic squamous cell carcinoma treated by $\mathrm{CO}_{2}$ TOLMS at a single academic institution.

\section{Materials and Methods}

This retrospective study included all patients treated by $\mathrm{CO}_{2}$ TOLMS and not previously treated for Tis-T2 glottic squamous cell carcinoma at the Department of OtorhinolaryngologyHead and Neck Surgery of the University of Brescia, Italy, from January 1988 to December 2018. All patients were included since the early adoption of this technique at our hospital to better evaluate the impact of the related learning curve. Data were collected by staff members and senior residents through retrospective clinical charts review, reevaluation of recorded videoendoscopies and radiologic imaging, assessment of follow-up documentation, and phone interview. Clinical charts with insufficient information for accurate staging $(\mathrm{n}=62)$ and/or lack of evaluation on postoperative outcomes $(\mathrm{n}=82)$ were excluded from the study, which was mainly focused on long-term DSS and OP, thus estimating the impact of different salvage therapeutic approaches during the course of the disease.

Data were recorded in a single database, regarding patient characteristics, endoscopic and imaging evaluation, tumor staging, type of surgery, recurrences, and follow-up. Redo $\mathrm{CO}_{2}$ TOLMS for positive margins (R1) was considered part of the primary treatment since it was usually performed within 45 days from the index procedure.

All patients were preoperatively examined under local anesthesia with videolaryngostroboscopy (Kay Digital Strobe 9200; Kay Elemetrics Corporation) and transnasal flexible videoendoscopy (Olympus Medical System Corporation) with white light and narrow band imaging (implemented after June 2007). In selected patients (cT1b with anterior commissure involvement to T2), preoperative computed tomography or magnetic resonance was performed to exclude involvement of the laryngeal framework and/or visceral (paraglottic and preepiglottic) spaces. Magnetic resonance has been the primary imaging choice at our institution since 2005. The examination was performed following a specific protocol for laryngeal cancer, with fast sequences and surface coils directly applied to the neck.

Microlaryngoscopy was performed under general anesthesia and muscle paralysis. A laser-safe tube (Laser-Shield; Medtronic Xomed) with an internal diameter not wider than $6.0 \mathrm{~mm}$ was inserted. The widest laryngoscope able to guarantee satisfactory glottic exposure was chosen ${ }^{5-7}$ and always suspended with the Boston University Suspension System (Pilling), which was employed to obtain a true suspension laryngoscopy, producing elevated vector forces on the laryngoscope. ${ }^{8}$ Intraoperative rigid endoscopy by $0^{\circ}$ and $70^{\circ}$ telescopes (Karl Storz) with white light and narrow band imaging was accomplished during microlaryngoscopy to reassess and better delineate the superficial resection margins. Clinical Tis-T1a lesions underwent an excisional biopsy approach, ${ }^{9,10}$ while larger cT1b-T2 were submitted to preoperative biopsy under local or general anesthesia.
Transoral resections were performed by an en bloc technique (type I-IV cordectomies according to the European Laryngological Society classification) ${ }^{11}$ or multibloc technique (type V or VI), depending on factors such as laryngeal exposure, tumor extension, site, and size. Surgical specimens were oriented at the end of the procedure by marking the superior edge with blue ink, thus allowing the dedicated head and neck surgical pathologist to easily identify the other superficial margins after appropriate definition of the type and side of cordectomy performed. In case of multibloc resections, a schematic drawing representing the 3-dimensional orientation of the inked surgical specimens was added to help its proper evaluation. Frozen sections were not routinely employed according to our institutional protocol. In extensive resections and in case of margins deemed insufficient at the intraoperative evaluation, extramargin resections were performed and the adjunctive specimens sent for definitive histopathology. Their negativity was thus considered indicative of an $\mathrm{R} 0$ resection.

The study was performed following the principles of the Declaration of Helsinki and was approved by the Research Review Board, Ethics Committee, of the ASST Spedali Civili of Brescia, Italy.

\section{Statistical Analysis}

Statistical analysis was carried out with Stata software version 13 (StataCorp). Overall survival, DSS, and OP rates were calculated with the Kaplan-Meier method, with events defined as death for every cause, death for laryngeal cancer, and total laryngectomy, respectively. For each survival estimate, the entry time was the date of conclusion of adjuvant treatment (if any) or date of surgery (for those not receiving further treatments). Event-free cases were censored at the last follow-up examination. Survival analysis was performed by univariate models based on log-rank test or Cox proportional hazard model, as appropriate. Hazard ratios were expressed as lifetime risks.

\section{Results}

A total of 1188 patients were included in the present retrospective analysis. The male:female ratio was 11.2:1, and the mean age was 64 years (range, 31-95). Patients were current or former smokers at the time of surgery in $92 \%$ of cases. Mean follow-up was 105 months (range, 1-350). Overall, 963 (81\%) patients reached the 5-year time point. Furthermore, $440(37 \%)$ had at least 10 years of follow-up and $71(6 \%)$ at least 20.

Distribution of $\mathrm{T}$ categories was as follows: $124(10 \%)$ Tis, 646 (54\%) T1a, 172 (15\%) T1b, and 246 (21\%) T2. No patient had lymph nodes or distant metastases at presentation. Distribution of types of cordectomies according to the European Laryngological Society classification was type I in 83, type II in 381 , type III in 247 , type IV in 93 , type V in 377 , and type VI in 7. Resection of a second lesion on the contralateral vocal fold (ie, for treatment of multifocal Tis or $\mathrm{T} 1 \mathrm{~b}$ without anterior commissure involvement) was also performed in 92 patients. 

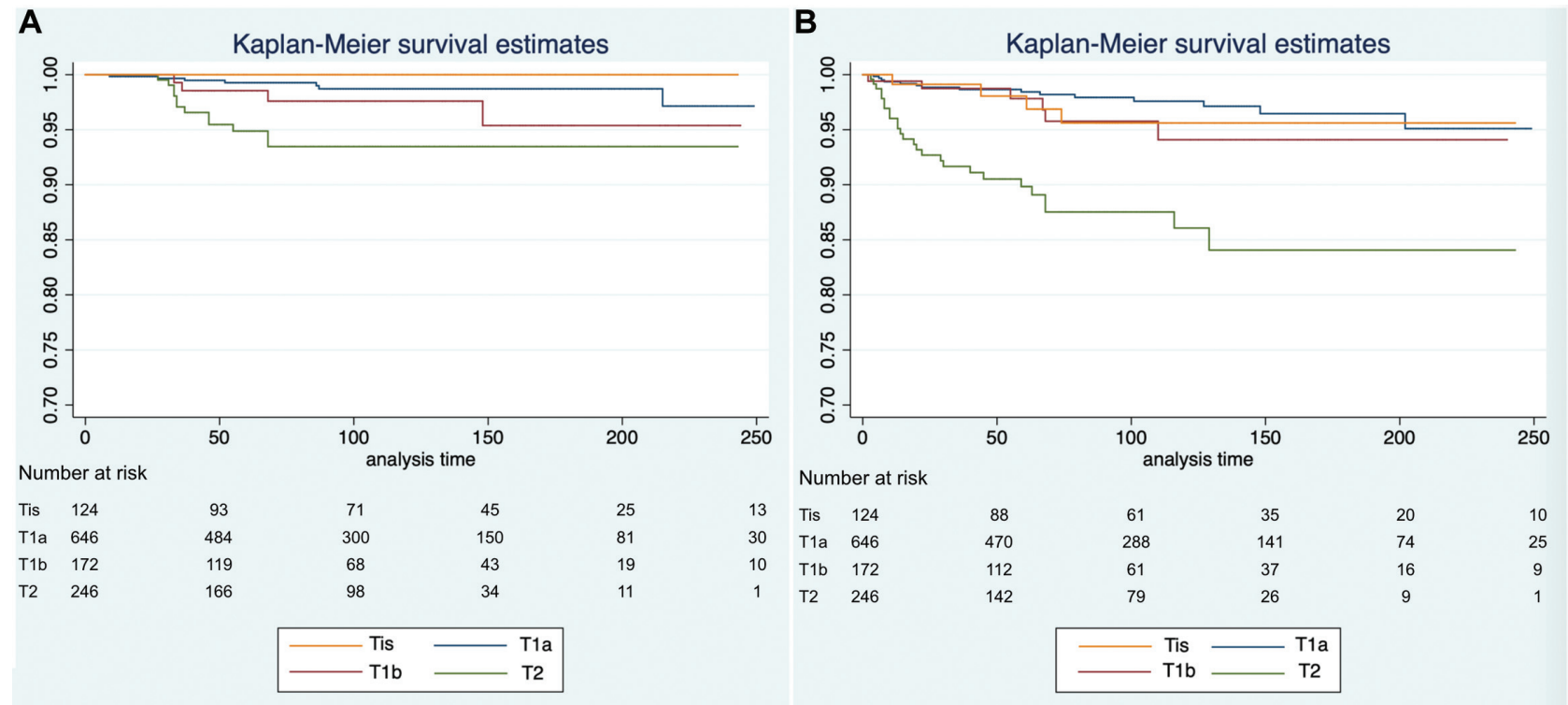

Figure I. Kaplan-Meier curves showing (A) disease-specific survival and (B) organ preservation rates for different pT categories.

Table I. DSS and OP Rates According to Disease Staging.

\begin{tabular}{|c|c|c|c|c|}
\hline & \multirow[b]{2}{*}{ No. (\%) } & \multicolumn{3}{|c|}{ DSS (95\% Cl), \% } \\
\hline & & $5 y$ & $10 y$ & $20 y$ \\
\hline $\mathrm{Tla}$ & $646(14.5)$ & $99.2(98.1-99.7)$ & 98.7 (97.1-99.4) & 97.1 (91.3-99.1) \\
\hline TIb & 172 (20.7) & $98.6(94.3-99.6)$ & $97.6(92.7-99.2)$ & $95.4(86.5-98.5)$ \\
\hline & No. (\%) & & OP (95\% Cl), \% & \\
\hline Carcinoma in situ & $124(10.4)$ & 98.1 (92.4-99.5) & $95.6(88.6-98.3)$ & $95.6(88.6-98.3)$ \\
\hline Tla & $646(14.5)$ & 98.4 (97-99.2) & $97.6(95.7-98.7)$ & $95.1(90.3-97.5)$ \\
\hline TIb & $172(20.7)$ & $97.8(93.3-99.3)$ & 94.1 (86.7-97.4) & 94.1 (86.7-97.4) \\
\hline $\mathrm{T} 2$ & $246(10.4)$ & $89.8(84.8-93.3)$ & 86.1 (79.4-90.7) & 84.1 (76.I-89.6) \\
\hline
\end{tabular}

Abbreviations: DSS, disease-specific survival; OP, organ preservation.

For the entire cohort, 10 - and 20 -year overall survival rates were $76.4 \%$ and $42.8 \%$, respectively. The 10 - and 20 year DSS rates were $97.6 \%$ and $96.3 \%$. The 10 - and 20 -year OP rates were $94.7 \%$ and $93 \%$. For DSS, T categories adequately stratified long-term survival, with an incremental risk of death going from Tis to T2 tumors. In particular, 10and 20 -year survival rates were both $100 \%$ for Tis, $99 \%$ and $97 \%$ for $\mathrm{T} 1 \mathrm{a}, 98 \%$ and $95 \%$ for $\mathrm{T} 1 \mathrm{~b}$, and both $93 \%$ for $\mathrm{T} 2$ (Figure IA, Table I).

For OP rates, it was possible to observe a bimodal distribution, with optimal results in Tis, T1a, and T1b and significantly worse laryngeal preservation for $\mathrm{T} 2$ tumors $(P=$ .009). Specifically, 10- and 20-year OP rates were both $96 \%$ for Tis, $98 \%$ and $95 \%$ for T1a, both $94 \%$ for T1b, and $86 \%$ and $84 \%$ for T2 (Figure IB, Table I).

During the study time frame, $91 \%$ of patients were treated by $\mathrm{CO}_{2}$ TOLMS alone, while the remaining needed adjunctive treatments (RT, chemotherapy, chemoradiotherapy, OPHL, or total laryngectomy) for disease control - specifically, $12 \%$ of Tis, $5 \%$ of T1a, $11 \%$ of T1b, and $20 \%$ of T2 $(P<$ $.001)$. Total laryngectomy was performed in $3 \%$ of Tis, $2 \%$ of T1a, $5 \%$ of T1b, and $10 \%$ of T2 $(P<.001)$.

In terms of the date of surgery (first 5 years [1988-1992] vs last 25 [1993-2018]), no significant difference in DSS and OP rates was observed between the groups, thus confirming the negligible impact of the learning curve on survival of EIGSCC. The same was true when the first 15 years (19882002 , in which only 1 surgeon [G.P.] was in charge of $\mathrm{CO}_{2}$ TOLMS at our department) were compared with the last 15 (2003-2018, in which 3 other surgeons [C.P., F.D.B., and A.P.] started working in this field).

For the impact of multiple sessions of $\mathrm{CO}_{2}$ TOLMS, DSS showed no significant difference among patients sequentially treated by 1,2 , or $>2$ procedures (Figure 2A, Table 2 ). 

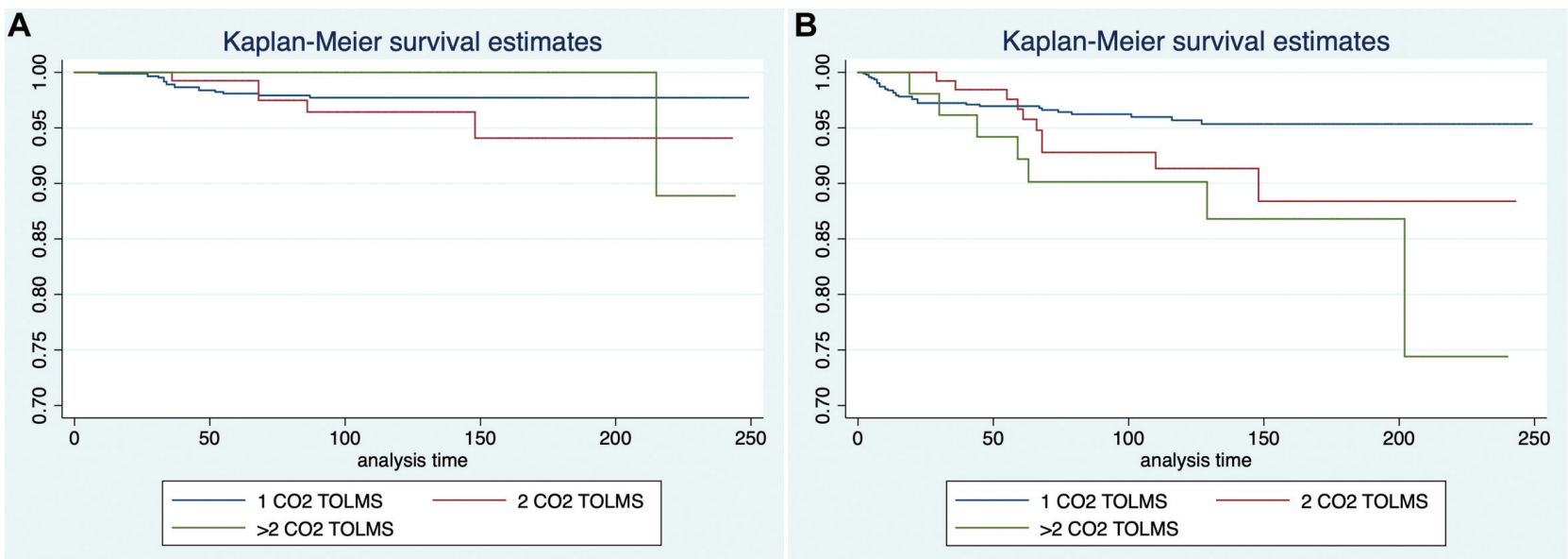

Figure 2. Kaplan-Meier curves showing (a) disease-specific survival and (b) organ preservation rates according to the number of $\mathrm{CO}_{2}$ TOLMS procedures. $\mathrm{CO}_{2}$ TOLMS, carbon dioxide transoral laser microsurgery.

Table 2. DSS and OP Rates According to the Number of $\mathrm{CO}_{2}$ TOLMS Procedures.

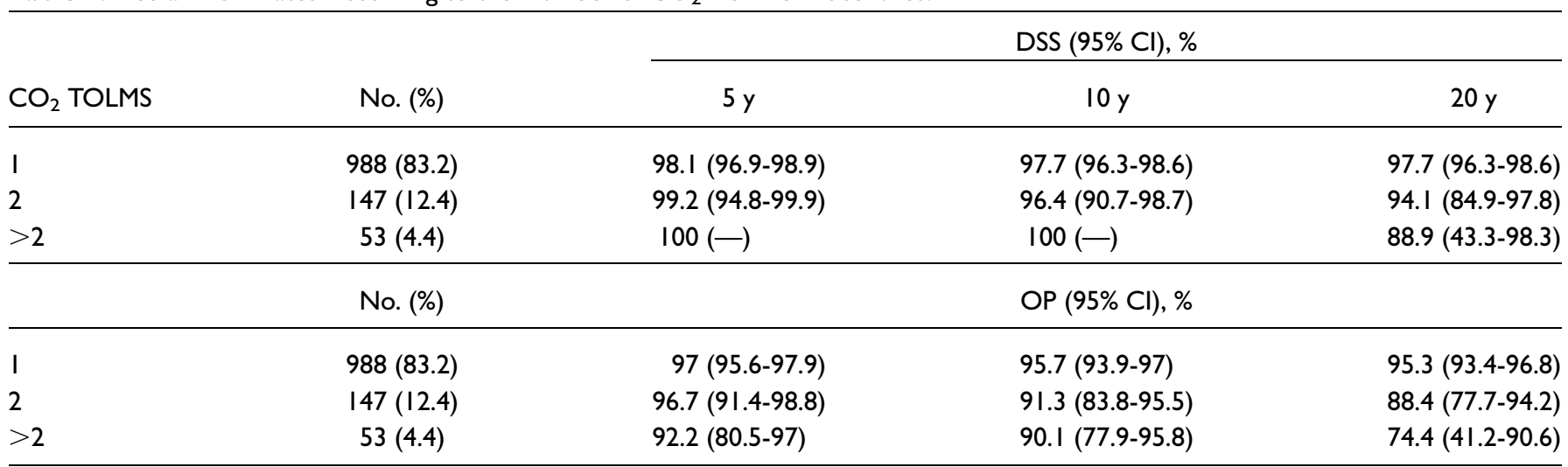

Abbreviations: $\mathrm{CO}_{2}$ TOLMS, carbon dioxide transoral laser microsurgery; DSS, disease-specific survival; OP, organ preservation.

Conversely, patients treated by $>2$ sessions of $\mathrm{CO}_{2}$ TOLMS showed a significantly worse OP rate $(P=.008)$ with a hazard ratio of 3 (Figure 2B).

Regarding the influence of the type of treatment in patients developing recurrence after transoral surgery, it was possible to observe an opposite trend between DSS and OP. In fact, patients salvaged by (chemo)radiotherapy had a significantly lower DSS $(P=.008$; hazard ratio, 6.6$)$ when compared with OPHL and repeat $\mathrm{CO}_{2}$ TOLMS (Figure 3B), while OPHL led to a lower chance of final OP $(P=.047$; hazard ratio, 3.5) when compared with (chemo)radiotherapy and redo $\mathrm{CO}_{2}$ TOLMS (Figure 3B). In both cases, patients amenable to salvage $\mathrm{CO}_{2}$ TOLMS had optimal results in terms of DSS and OP rates.

\section{Discussion}

Our series provides an oncologic long-term validation of $\mathrm{CO}_{2}$ TOLMS as an ideal treatment strategy for EIGSCC. While long-term overall survival did not prove to be strictly correlated with such a procedure, since it was mainly influenced by patients' advanced age, DSS and OP were well over $90 \%$ at 10 and 20 years of follow-up, with the exception of OP in T2 glottic squamous cell carcinoma. The minimally invasive transoral approach herein considered, in fact, proved to be a repeatable procedure, maintaining optimal DSS even after multiple interventions. In line with such an assumption, OP was significantly reduced only in patients needing $>2$ transoral operations. In patients needing salvage treatment after $\mathrm{CO}_{2}$ TOLMS, a revision transoral resection provided optimal results in terms of DSS and OP. Conversely, patients needing different laryngeal preservation treatments as salvage experienced significantly lower OP (OPHL) and DSS ([chemo]radiotherapy).

To the best of our knowledge, this is the largest series assessing long-term outcomes of $\mathrm{CO}_{2}$ TOLMS for EIGSCC to date. Study endpoints were selected to provide accurate information on the disease course in relation to the characteristics of EIGSCC while minimizing the risk of bias. In fact, local relapse represents the main mode of failure, and multiple transoral resections may successfully achieve longterm local control without compromising survival. In this view, DSS provided an overall measure of the disease course 

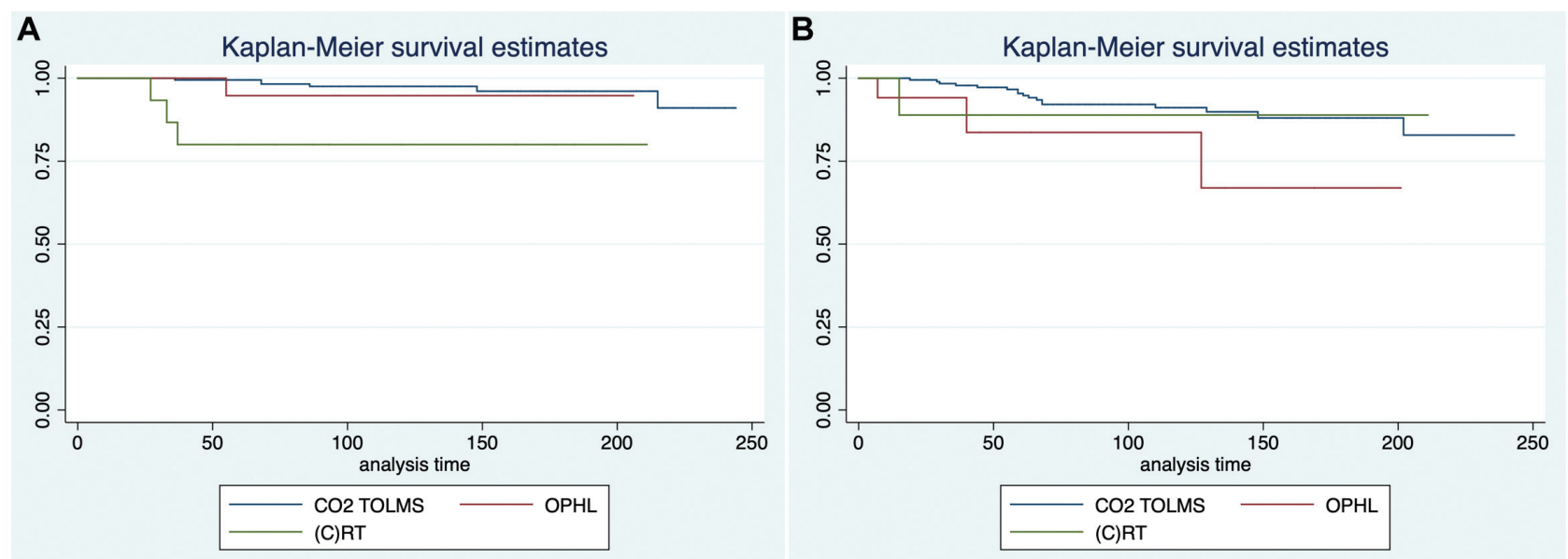

Figure 3. Kaplan-Meier curves showing (A) disease-specific survival and (B) organ preservation rates in patients with recurrence according to the type of salvage surgical procedure. $\mathrm{CO}_{2}$ TOLMS, carbon dioxide transoral laser microsurgery; (C)RT, (chemo)radiotherapy; OPHL, open partial horizontal laryngectomy.

and the probability of long-term control obtained by $\mathrm{CO}_{2}$ TOLMS with eventual adjunctive procedures. This allowed us to take into account the chance of disease control by a single surgical procedure, that of salvage after single or multiple recurrences, and the impact of combined treatment strategies. However, it is essential to consider that multiple local procedures may lead to progressive functional impairment and a higher risk of recurrence involving the laryngeal framework, ultimately influencing OP. Therefore, this endpoint should be considered an accurate indicator of the chance of conservative disease management.

Reports assessing oncologic outcomes of $\mathrm{CO}_{2}$ TOLMS in EIGSCC are progressively increasing in the literature, as described in a recent article by Mendelsohn et al. ${ }^{12}$ This has been favored by the first descriptions of the oncologic validity of this procedure by Steiner and coworkers, ${ }^{13}$ as well as by the introduction of the European Laryngological Society classification of $\mathrm{CO}_{2}$ TOLMS cordectomies. ${ }^{11}$ In fact, the dissemination of such a well-defined classification scheme optimized, in the last 20 years, the chance to collect and compare outcomes in a standardized manner. Thanks to the numerous series now present in the international literature, $\mathrm{CO}_{2}$ TOLMS is currently considered a valid treatment option for laryngeal cancer even in the absence of highquality randomized clinical trials comparing it with alternatives (ie, RT and OPHL). In particular, several systematic reviews and meta-analyses have confirmed its noninferiority as compared with RT for EIGSCC, in terms of oncologic and functional outcomes, ${ }^{14-20}$ with a trend toward better results in $\mathrm{CO}_{2}$ TOLMS. However, because of the relatively recent widespread use of $\mathrm{CO}_{2}$ TOLMS, there is a paucity of information on long-term oncologic and OP results.

A typical concern in consideration of the minimally invasive nature of this type of surgery, aimed at preserving the majority of laryngeal mucosa, is the potential risk of tobacco-related field cancerization. Notwithstanding, $\mathrm{CO}_{2}$ TOLMS is a repeatable procedure, and subsequent interventions may successfully manage local recurrences and second tumors. As confirmation of this evidence, our results showed stable DSS and OP rates even decades after primary treatment, thus demonstrating that the diffuse oncogenic effect of smoking does not impair survival and laryngeal preservation when a minimally invasive option has been chosen. In particular, even adjunctive $\mathrm{CO}_{2}$ TOLMS for relapse/second tumor did not significantly impair DSS. However, patients needing $>2$ procedures represented a high-risk subgroup with significantly lower OP, potentially reflecting a higher local aggressiveness of the primary tumor and/or a more pronounced tendency toward dysplastic/neoplastic transformation of the residual laryngeal mucosa.

These results confirm the importance of treating EIGSCC with a single-treatment modality, ${ }^{21}$ avoiding RT in case of persistence, recurrence, or second tumor still amenable to $\mathrm{CO}_{2}$ TOLMS. ${ }^{22}$ In this view, $\mathrm{CO}_{2}$ TOLMS should be regarded as a repeatable procedure, and patients should be carefully monitored according to dedicated protocols, including seriate biologic endoscopic and radiologic examinations, ${ }^{23-25}$ to avoid late diagnosis of recurrence/metachronous tumor in the presence of laryngeal framework involvement. ${ }^{26}$ Our data confirm that $\mathrm{CO}_{2}$ TOLMS, with adequate indications and patient selection, provides optimal oncologic results as a salvage procedure after previous transoral laser surgery, as already demonstrated by our group in the clinical setting of failure after $\mathrm{RT}^{27,28}$ In recurrent cases not manageable by $\mathrm{CO}_{2}$ TOLMS, it should be considered that OPHL, although associated with lower OP, resulted in a better DSS than RT. However, it is essential to take into account the potential bias of patient selection, since patients with heavier comorbidities may be more frequently directed to RT.

While other authors validated the efficacy of $\mathrm{CO}_{2}$ TOLMS in large monoinstitutional series, ${ }^{29-34}$ our long-term data provide confirmation of the absence of a significant outcome difference, at least concerning EIGSCC, related to the surgeons' learning curve. Moreover, in terms of long-term functional outcomes, a recent analysis by $\mathrm{Ma}$ et $\mathrm{al}^{35}$ reported better results with $\mathrm{CO}_{2}$ TOLMS than with RT. This may be 
related to the progressive tissue fibrosis that continues even years after primary irradiation, while vocal outcomes after $\mathrm{CO}_{2}$ TOLMS tend to stabilize after 6 months. ${ }^{36}$ Moreover, repercussions on quality of life seem to be limited to the 3month time point, ${ }^{37}$ since various phenomena of coping, adaptation, and rehabilitation may play a role in improving vocal performance with time. Finally, long-term general quality of life seems to be adequately maintained in patients treated by $\mathrm{CO}_{2}$ TOLMS, as demonstrated by Valls-Mateus et al, ${ }^{38}$ similar to what is seen in immediate posttreatment outcomes. ${ }^{39}$

These results may help to better guide the treatment for EIGSCC at the level of a multidisciplinary tumor board, choosing between $\mathrm{CO}_{2}$ TOLMS and RT on the basis of personalized evidence-based criteria and not standardized policies. In particular, while not directly related to treatment outcomes, patient preference should be carefully considered in the long-term management of the disease. In this regard, $\mathrm{CO}_{2}$ TOLMS is frequently reported as the patients' treatment of choice ${ }^{40-42}$ : this is of particular interest in consideration of its favorable cost-effectiveness ratio as compared with RT. ${ }^{43}$

The UK NICE guidelines (National Institute for Health and Care Excellence) advise transoral surgery for all T1a tumors, whereas $\mathrm{CO}_{2}$ TOLMS and RT are both suggested for T1b to T2 lesions. ${ }^{44}$ Similarly, according to the Dutch guidelines for treatment of laryngeal squamous cell carcinoma, $\mathrm{CO}_{2}$ TOLMS is the treatment of choice for superficial T1a midcord lesions, while for more extended T1 and T2 tumors, RT is still the most advocated treatment. ${ }^{45}$ Therefore, RT remains the most frequently applied treatment for EIGSCC in Northern Europe and United States, as demonstrated by a Surveillance, Epidemiology and End Results-based population study ${ }^{46}$ on 5333 patients treated by RT versus 1913 treated by surgery alone. In light of the long-term results that we present here, a similar validation of patients treated by RT is warranted to adequately compare its validity and fine-tune treatment indications for those affected by EIGSCC.

Concerning study limitations, this is a retrospective cohort that focused only on DSS and OP. Consequently, a number of confounding factors may have played a role in the choice of treatment and its oncologic results. However, these limitations are partially mitigated by the large number of patients, the selection of a specific subgroup of laryngeal cancer (ie, EIGSCC), and the precise definition of straightforward endpoints to be investigated.

\section{Conclusions}

Our series provides long-term insight of $\mathrm{CO}_{2}$ TOLMS as a treatment strategy for EIGSCC. In particular, DSS was not significantly reduced even after multiple procedures. A decline in the OP rate was observed only in patients needing $>2 \mathrm{CO}_{2}$ TOLMS procedures. Finally, revision $\mathrm{CO}_{2}$ TOLMS provided optimal results in terms of DSS and OP even in patients with recurrence after prior transoral surgery.

\section{Author Contributions}

Cesare Piazza, study design, drafting of the manuscript, revision of the manuscript, final approval, final agreement; Alberto Paderno, study design, drafting of the manuscript, revision of the manuscript, final approval, final agreement; Francesca Del Bon, study design, drafting of the manuscript, final approval, final agreement; Davide Lancini, study design, drafting of the manuscript, final approval, final agreement; Milena Fior, study design, drafting of the manuscript, final approval, final agreement; Giulia Berretti, study design, drafting of the manuscript, final approval, final agreement; Paolo Bosio, study design, drafting of the manuscript, final approval, final agreement; Alberto Deganello, study design, drafting of the manuscript, final approval, final agreement; Giorgio Peretti, study design, drafting of the manuscript, revision of the manuscript, final approval, final agreement.

\section{Disclosures}

Competing interests: None.

Sponsorships: None.

Funding source: None.

\section{References}

1. Remacle M, Arens C, Eldin MB, et al. Laser-assisted surgery of the upper aero-digestive tract: a clarification of nomenclature. A consensus statement of the European Laryngological Society. Eur Arch Otorhinolaryngol. 2017;274(10):3723-3727.

2. Succo G, Peretti G, Piazza C, et al. Open partial horizontal laryngectomies: a proposal for classification by the working committee on nomenclature of the European Laryngological Society. Eur Arch Otorhinolaryngol. 2014;271(9):2489-2496.

3. Warner L, Chudasama J, Kelly CG, et al. Radiotherapy versus open surgery versus endolaryngeal surgery (with or without laser) for early laryngeal squamous cell cancer. Cochrane Database Syst Rev. 2014;(12):CD002027.

4. Ogol'tsova E, Paches A, Matiakin E, et al. Comparative evaluation of the effectiveness of radiotherapy, surgery and combined treatment of stage I-II laryngeal cancer (T1-2NoMo) based on the data of a cooperative randomized study. Vestn Otorinolaringol. 1990;(3):3-7.

5. Piazza C, Mangili S, Bon FD, et al. Preoperative clinical predictors of difficult laryngeal exposure for microlaryngoscopy: the Laryngoscore. Laryngoscope. 2014;124(11):2561-2567.

6. Piazza C, Paderno A, Grazioli P, et al. Laryngeal exposure and margin status in glottic cancer treated by transoral laser microsurgery. Laryngoscope. 2018;128(5):1146-1151.

7. Incandela F, Paderno A, Missale F, et al. Glottic exposure for transoral laser microsurgery: proposal of a mini-version of the laryngoscore. Laryngoscope. 2019;129(7):1617-1622.

8. Zeitels SM, Burns JA, Dailey SH. Suspension laryngoscopy revisited. Ann Otol Rhinol Laryngol. 2004;113(1):16-22.

9. Blakeslee D, Vaughan CW, Shapshay SM, Simpson GT, Strong MS. Excisional biopsy in the selective management of T1 glottic cancer: a three-year follow-up study. Laryngoscope. 1984;94(4):488-494. 
10. Peretti G, Cappiello J, Nicolai P, Smussi C, Antonelli AR Endoscopic laser excisional biopsy for selected glottic carcinomas. Laryngoscope. 1994;104(10):1276-1279.

11. Remacle M, Eckel HE, Antonelli A, et al. Endoscopic cordectomy: a proposal for a classification by the Working Committee, European Laryngological Society. Eur Arch Otorhinolaryngol. 2000;257(4):227-231.

12. Mendelsohn AH, Remacle MJ. Vocal fold cancer transoral laser microsurgery following European Laryngological Society laser cordectomy classification. Front Oncol. 2018;8:231.

13. Harris AT, Tanyi A, Hart RD, et al. Transoral laser surgery for laryngeal carcinoma: has Steiner achieved a genuine paradigm shift in oncological surgery? Ann R Coll Surg Engl. 2018; 100(1):2-5.

14. Ding Y, Wang B. Efficacy of laser surgery versus radiotherapy for treatment of glottic carcinoma: a systematic review and meta-analysis. Lasers Med Sci. 2019;34(5):847-854.

15. Gioacchini FM, Tulli M, Kaleci S, Bondi S, Bussi M, Re M. Therapeutic modalities and oncologic outcomes in the treatment of T1b glottic squamous cell carcinoma: a systematic review. Eur Arch Otorhinolaryngol. 2017;274(12):4091-4102.

16. Guimaraes AV, Dedivitis RA, Matos LL, Aires FT, Cernea CR. Comparison between transoral laser surgery and radiotherapy in the treatment of early glottic cancer: a systematic review and meta-analysis. Sci Rep. 2018;8(1):11900.

17. Hendriksma M, Heijnen BJ, Sjogren EV. Oncologic and functional outcomes of patients treated with transoral CO2 laser microsurgery or radiotherapy for $\mathrm{T} 2$ glottic carcinoma: a systematic review of the literature. Curr Opin Otolaryngol Head Neck Surg. 2018;26(2):84-93.

18. Mo HL, Li J, Yang X, et al. Transoral laser microsurgery versus radiotherapy for $\mathrm{T} 1$ glottic carcinoma: a systematic review and meta-analysis. Lasers Med Sci. 2017;32(2):461-467.

19. Vaculik MF, MacKay CA, Taylor SM, Trites JRB, Hart RD, Rigby MH. Systematic review and meta-analysis of T1 glottic cancer outcomes comparing $\mathrm{CO} 2$ transoral laser microsurgery and radiotherapy. J Otolaryngol Head Neck Surg. 2019;48(1):44.

20. Warner L, Lee K, Homer JJ. Transoral laser microsurgery versus radiotherapy for T2 glottic squamous cell carcinoma: a systematic review of local control outcomes. Clin Otolaryngol. 2017;42(3):629-636.

21. Baird BJ, Sung CK, Beadle BM, Divi V. Treatment of earlystage laryngeal cancer: a comparison of treatment options. Oral Oncol. 2018;87:8-16.

22. Lucioni M, Bertolin A, Lionello M, Giacomelli L, Rizzotto G, Marioni G. Salvage transoral laser microsurgery for recurrent glottic carcinoma after primary laser-assisted treatment: analysis of prognostic factors. Head Neck. 2016;38(7):1043-1049.

23. Gallet P, Rumeau C, Nguyen DT, Teixeira PA, Baumann C, Toussaint B. "Watchful observation" follow-up scheme after endoscopic $\mathrm{CO} 2$ laser treatment for small glottic carcinomas: a retrospective study of 93 cases. Clin Otolaryngol. 2017;42(6): 1193-1199.

24. Marchi F, Piazza C, Ravanelli M, et al. Role of imaging in the follow-up of T2-T3 glottic cancer treated by transoral laser microsurgery. Eur Arch Otorhinolaryngol. 2017;274(10):36793686 .
25. Simo R, Bradley P, Chevalier D, et al. European Laryngological Society: ELS recommendations for the follow-up of patients treated for laryngeal cancer. Eur Arch Otorhinolaryngol. 2014; 271(9):2469-2479.

26. Horwich P, Rigby MH, MacKay C, et al. Laryngeal recurrence sites in patients previously treated with transoral laser microsurgery for squamous cell carcinoma. J Otolaryngol Head Neck Surg. 2018;47(1):14.

27. Puxeddu R, Piazza C, Mensi MC, Ledda GP, Argiolas F, Peretti G. Carbon dioxide laser salvage surgery after radiotherapy failure in T1 and T2 glottic carcinoma. Otolaryngol Head Neck Surg. 2004;130(1):84-88.

28. Del Bon F, Piazza C, Mangili S, Redaelli De Zinis LO, Nicolai P, Peretti G. Transoral laser surgery for recurrent glottic cancer after radiotherapy: oncologic and functional outcomes. Acta Otorhinolaryngol Ital. 2012;32(4):229-237.

29. Vilaseca I, Nogues-Sabate A, Aviles-Jurado FX, et al. Factors of local recurrence and organ preservation with transoral laser microsurgery in laryngeal carcinomas: CHAID decision-tree analysis. Head Neck. 2019;41(3):756-764.

30. Ansarin M, Cattaneo A, De Benedetto L, et al. Retrospective analysis of factors influencing oncologic outcome in 590 patients with early-intermediate glottic cancer treated by transoral laser microsurgery. Head Neck. 2017;39(1):71-81.

31. Canis M, Ihler F, Martin A, Matthias C, Steiner W. Transoral laser microsurgery for T1a glottic cancer: review of 404 cases. Head Neck. 2015;37(6):889-895.

32. Canis M, Martin A, Ihler F, et al. Transoral laser microsurgery in treatment of pT2 and pT3 glottic laryngeal squamous cell carcinoma — results of 391 patients. Head Neck. 2014;36(6):859866.

33. Ding S, Huang J, Huang Z, Xu H, Guo W, Zhang Y. Variation in prognosis of early laryngeal carcinoma after different types of cordectomy with transoral laser microsurgery. Acta Otolaryngol. 2018;138(8):741-745.

34. Peretti G, Piazza C, Cocco D, et al. Transoral CO(2) laser treatment for $\mathrm{T}(\mathrm{is})-\mathrm{T}(3)$ glottic cancer: the University of Brescia experience on 595 patients. Head Neck. 2010;32(8):977-983.

35. Ma Y, Green R, Pan S, McCabe D, Goldberg L, Woo P. Longterm voice outcome following radiation versus laser microsurgery in early glottic cancer. J Voice. 2019;33(2):176-182.

36. Hendriksma M, van Loon Y, Klop WMC, et al. Quality of life and voice outcome of patients treated with transoral $\mathrm{CO} 2$ laser microsurgery for early glottic carcinoma (T1-T2): a 2-year follow-up study. Eur Arch Otorhinolaryngol. 2019;276(3):805814.

37. Bui AT, Yong Ji KS, Pham CT, Le KM, Tong TX, Lee WT. Longitudinal evaluation of quality of life in laryngeal cancer patients treated with surgery. Int J Surg. 2018;58:65-70.

38. Valls-Mateus M, Ortega A, Blanch JL, Sabater F, BernalSprekelsen M, Vilaseca I. Long-term quality of life after transoral laser microsurgery for laryngeal carcinoma. J Surg Oncol. 2016;114(7):789-795.

39. Kono T, Saito K, Yabe H, Uno K, Ogawa K. Comparative multidimensional assessment of laryngeal function and quality of life after radiotherapy and laser surgery for early glottic cancer. Head Neck. 2016;38(7):1085-1090. 
40. McNeil ML, Wilke DR, Taylor SM. Patient preferences in early glottic cancer treatment. Ear Nose Throat J. 2016;95(7):281-283.

41. van Loon Y, Hendriksma M, Langeveld TPM, de Jong MA, Baatenburg de Jong RJ, Sjogren EV. Treatment preferences in patients with early glottic cancer. Ann Otol Rhinol Laryngol. 2018;127(3):139-145.

42. Zahoor T, Dawson R, Sen M, Makura Z. Transoral laser resection or radiotherapy? Patient choice in the treatment of early laryngeal cancer: a prospective observational cohort study. J Laryngol Otol. 2017;131(6):541-545.

43. Prettyjohns M, Winter S, Kerawala C, Paleri V. Transoral laser microsurgery versus radiation therapy in the management of $\mathrm{T} 1$ and T2 laryngeal glottic carcinoma: which modality is costeffective within the UK? Clin Otolaryngol. 2017;42(2):404-415.
44. National Institute for Clinical Excellence. Cancer of the upper aerodigestive tract: assessment and management in people aged 16 and over. Accessed December 7, 2019. http://www.nice.org .uk/NG36

45. Nederlandse Werkgroep Hoofd Halstumoren. Richtlijn Larynxcarcinomen Kwaliteitsintistuut voor de Gezondheidszorg CBO. Accessed December 7, 2019. http://www.kwaliteitskoepel.nl/ assets/structured-files/2011/Larynxcarcinoom.pdf

46. Zhan C, Yang X, Song X, Yan L. Radiotherapy vs surgery for T1-2N0M0 laryngeal squamous cell carcinoma: a populationbased and propensity score matching study. Cancer Med. 2018; 7(7):2837-2847. 